\title{
Tangence
}

\section{Pourquoi je n'écris pas d'essais postmodernes}

\section{Pierre Milot}

Numéro 39, mars 1993

La fiction postmoderne

URI : https://id.erudit.org/iderudit/025756ar

DOI : https://doi.org/10.7202/025756ar

Aller au sommaire du numéro

Éditeur(s)

Tangence

ISSN

0226-9554 (imprimé)

1710-0305 (numérique)

Découvrir la revue

Citer cet article

Milot, P. (1993). Pourquoi je n'écris pas d'essais postmodernes. Tangence, (39), 102-111. https://doi.org/10.7202/025756ar d'utilisation que vous pouvez consulter en ligne.

https://apropos.erudit.org/fr/usagers/politique-dutilisation/ 


\section{Pourquoi je n'écris pas d'essais postmodernes}

\section{Pierre Milot}

On sait que la discussion sur le postmodernisme concerne, depuis le début, tout autant les écrivains et les essayistes que les philosophes: les uns s'en servent comme d'une catégorie esthétique inhérente à une certaine mise en forme de leur propre pratique d'écriture, les autres comme d'un nouveau paradigme capable de rendre compte de certaines tendances de la production littéraire et philosophique. Aussi, le concept de postmodernité ne fait pas consensus, pas plus dans sa définition concurrentielle (par rapport au concept de modernité et à celui d'avant-garde) que dans ses conditions de possibilité: pour les uns, il faut le resituer dans une historicité bien délimitée, pour les autres, c'est précisément cette opération qui demeure impensable. Sans compter que la discussion sur la postmodernité s'est inscrite dans différents champs disciplinaires (dont la philosophie et la littérature) et a fait appel à la notion de genre (dont l'essai et le roman).

L'argument du présent essai consistera donc à reconsidérer le postmodernisme dans sa problématisation disciplinaire et dans son effectuation au sein de différents genres, et ce, à partir de certains de ses principaux protagonistes. Qu'il s'agisse des textes qui sont à l'origine du débat parisien, et dont j'ai déjà parlé ailleurs ${ }^{1}$, ou encore de ceux qui l'avaient précédé et qui l'ont suivi, il s'agira de comparer la façon dont des écrivains, des essayistes et des philosophes ont contribué à mener ce débat à partir de leur champ disciplinaire respectif et en fonction du genre pratiqué. Il faudra par conséquent s'interroger sur les conditions d'exercice d'une discussion où les conditions institutionnelles des termes de l'échange sont demeurées la plupart du temps méconnues, c'est-à-dire où la division institutionnelle du travail intellectuel est restée dans sa boite noire. D'où une réflexion cherchant à clarifier l'ordre des institutions et

1 Cf. Pierre Milot, *Modernité, avant-garde, postmodernité. Les conditions du débat ", dans La camera obscura du postmodernisme, Montréal, l'Hexagone, coll. * Essais littéraires *, 1988, p. 61-83. 
des discours: comprendre en quoi ceux qui se disputent leur part du marché intellectuel ne partagent pas les mêmes principes de discussion, ne respectent pas la même logique argumentative, n'utilisent pas les mêmes procédés rhétoriques et ne visent pas les mêmes fins éthiques et esthétiques. Tout compte fait, et toutes choses étant égales, donner à voir que le postmodernisme peut relever de la fiction théorique d'une part, et de la littérophilosophie d'autre part, et en donner à lire les conséquences pour la fiction comme pour la théorie, pour la littérature comme pour la philosophie.

\section{Hermann Broch selon Guy Scarpetta et Hannah Arendt: deux poids, deux mesures}

Pour amorcer notre discussion par un cas de figure, examinons la façon dont Hermann Broch est devenu, par l'entremise de Guy Scarpetta, un écrivain postmoderne par anticipation, alors qu'il était au contraire, pour Hannah Arendt, l'écrivain de son époque le plus apte à générer une théorie de la littérature arrimée à la théorie de la connaissance et à l'éthique ${ }^{2}$.

Dans la section “Écritures" de L'impureté3, Scarpetta propose une "Introduction à Hermann Broch", elle-même précédée d'un préambule où l'on apprend d'emblée que "plus qu'aucune autre partie de ce livre, peut-être, celle-ci sera subjective et lacunaire. Subjective: parce que c'est en écrivain, ici, que j'aborde certains livres et certaines œuvres, et que c'est en écrivain que je les lis." Et il ajoute, pour tenter de lever tout malentendu possible envers cette façon d'aborder les livres et les œuvres en question: "moins pour les poser en objets de savoir, moins pour produire, à leur propos, un effet de connaissance objective, que pour y retrouver, voire y solliciter, l'écho de mes propres préoccupations, dont je ne saurais m'abstraire" (p. 231). Je ne puis ici m'empêcher de faire remarquer que cette précaution liminaire, si elle a pour elle la légitimité de l'écrivain qui parle en toute subjectivité, est un

2 Il faut noter que Jean-Marie Schaeffer, d'une façon qui ne nous intéresse pas ici, a cherché à analyser les rapports entre théorie littéraire, théorie des genres et théorie de la connaissance, dans son essai intitulé * Du texte au genre. Notes sur une problématique générique", dans Théorie des genres, Gérard Genette et al., Paris, Seuil. coll. •Points*, 1986, p. 179-205.

3 Guy Scarpetta, L'impureté, Paris, Grasset, coll. •Figures *, 1985. 
procédé rhétorique typique d'une certaine posture postmoderne envers la théorie. Nous y reviendrons. Mais retenons, pour l'instant, que là où les choses se compliquent, c'est quand dans la conclusion de ce même préambule Scarpetta s'aventure à traiter des rapports entre littérature et philosophie en affirmant que le roman est désormais * capable de traiter, mieux que toute philosophie, la raison irrationnelle qui caractérise l'époque", que le roman a "avalé" le discours philosophique et enfin que "le discours philosophique n'est ni l'envers du roman, ni son métalangage; au contraire: il n'en est qu'un sous-ensemble, un matériau possible." Et c'est sans compter que *le roman, lui (telle est du moins la conviction qui s'est peu à peu imposée à moi), par sa capacité à tout absorber, pourrait bien être désormais en mesure de devenir le métalangage absolu" (p. 233). En trois pages, et sans s'en rendre compte, Scarpetta est passé de la faculté subjective du jugement de goût (selon Kant) à la catégorie objective d'une dialectique totalisante (selon Hegel).

Ce qu'il faut comprendre, c'est que c'est à travers ce motif de la supériorité absolue de la littérature sur la philosophie que Scarpetta entreprend son apologie de Broch et du roman: "Broch [...] perçoit l'impuissance du discours philosophique [...] d'où la nécessité du roman. Ou encore: "le roman peut ainsi confronter l'hyper-rationnel (inclusion de la réflexion intellectuelle) et l'hyper-lyrique (l'expression poétique ou mystique); il devient le dispositif d'intégration maximale, le comble du métalangage, apte à traiter tous les autres discours (philosophie comprise) comme des sous-ensembles" (p. 235). Mais Scarpetta avait eu un prédécesseur en la personne de Philippe Sollers qui, répondant à des questions de Jacques Henric ${ }^{4}$, en 1979, avait déclamé le même motif, sauf qu'au lieu de mettre la philosophie entre parenthèses ("philosophie comprise"), il faisait du "Socrate, en passant ": "Je parle de philosophie parce que le geste de la littérature est maintenant de montrer que le discours philosophique est intégrable à la position du sujet littéraire pour peu que son expérience soit menée jusqu'au bout de l'horizon transcendantal." Après l'ouverture andante du thème proposé, voilà que Sollers s'emporte et se propulse dans un allegro con brio: "Ce qui produit un renversement culturel considérable, à savoir que la

4 Philippe Sollers, "Socrate, en passant", dans Théorie des exceptions, Paris, Gallimard, coll. * Folio Essais „, 1986, p. 266-284. 
philosophie se trouve non pas niée, mais intégrée dans un discours tout simplement supérieur qui fait que, dans cette position d'énonciation, on peut à tout instant traiter les différents systèmes philosophiques qui ont eu lieu au cours des temps, comme un naturaliste vous montrerait des vertèbres " (p. 269). Rien de moins. Hegel plus Darwin? Sollers a prévu l'objection: "c'était l'idée de Hegel mais on peut mettre Hegel aussi dans ce que je suis en train d'évoquer ". D'autant plus que Balzac avait conçu sa Comédie bumaine "comme une société animale ". Donc conclusion du syllogisme intempestif: "l'écrivain est là pour [...] manifester [de] la crise, ou [de] la disparition, de la philosophie".

Mais Scarpetta a aussi son philosophe de service (doublé d'un romancier) pour "évoquer" sa "conviction" concernant "la fonction de connaissance de la littérature". C'est ainsi qu'on apprendra que Bernard-Henri Lévy avait "énoncé" (de sa "position d'énonciation ") qu'il n'était pas pour lui "de meilleure façon de saisir le processus contradictoire qui a mené au chaos de 1418 (et, au-delà, au triomphe des totalitarismes modernes) que de lire L'bomme sans qualités de Musil ". Scarpetta, lui, c'est dans Les somnambules de Broch qu'il a fait la même expérience. En somme, si on fait le compte, Sollers, Henric, Lévy et Scarpetta s'entendent tous pour "énoncer " (de leur "position d'énonciation" de romanciers) que le genre romanesque a imposé sa volonté de puissance au champ intellectuel. Ce qui ne va pas sans quelques incohérences. Ainsi, lorsque Scarpetta veut traiter des "modes d'intégration" de la narration littéraire et de l'essai philosophique dans Les somnambules, il souligne "la nécessité d'une intrusion d'auteur (car on ne peut plus, dès lors qu'il s'agit d'un essai et non d'un simple commentaire, se contenter de parler de narrateur" (p. 239). Mais "dès lors" que l'auteur s'introduit dans le roman selon le "registre plus intellectuel " de l'essai (par exemple, toute la dernière section du roman relève de la théorie de la connaissance), comment peut-on parler, comme le fait Scarpetta, d'un "montage de discours aux registres divers"? Puisque par ce relativisme discursif qui se refuse à toute normativité, on s'interdit "dès lors" de pouvoir traiter de la fonction éthique de la littérature, alors même que Scarpetta reconnaît qu'elle était au principe de l'entreprise romanesque de Broch.

Et c'est d'abord et avant tout cela que la philosophe Hannah Arendt appelait *l'exigence éthique " chez Hermann Broch, tant 
106

chez le romancier que chez l'essayiste. Dans son introduction à Création littéraire et connaissances (introduction dont Scarpetta ne fait mention que dans une note en bas de page), Hannah Arendt présente Broch par la phrase suivante: "Hermann Broch fut un écrivain malgré lui. Être écrivain sans vouloir l'être, voilà le trait fondamental de son caractère, voilà ce qui inspira l'action dramatique de son ouvre maitresse et devint le principal conflit de sa vie " ( $p$. 7). Et il ne s'agit pas là d'un simple énoncé provocateur ou d'une redondante rhétorique de la subversion. Toute l'introduction consiste en fait à démontrer cette thèse de départ en prenant à témoin la trajectoire intellectuelle et les livres de Broch, tant ses romans que ses essais. Ce dont elle cherche à rendre compte, c'est du conflit permanent qui a hanté Broch, à savoir la tension constante entre création littéraire, connaissance et action. Et la conclusion à laquelle elle en arrive est que "sur le plan de la création et du travail il s'était, dans les dernières années de son existence, totalement convaincu de la primauté de la connaissance sur la création littéraire, de la science sur l'art et finalement même d'une sorte de priorité, sans qu'il s'agisse d'une primauté, d'une théorie générale de la connaissance sur la science et la politique" (p. 11).

Par contre, Hannah Arendt nous prévient qu'il ne s'agit pas pour autant de faire de Hermann Broch un philosophe et de ses essais des œuvres philosophiques: il reste un écrivain pour qui la philosophie et la théorie de la connaissance sont devenues des activités inhérentes à son travail: "De la distance qui le sépare de la philosophie, Broch a toujours été conscient", dit-elle. Par exemple, dans ses premiers livres et jusqu'à son étude sur Hugo von Hofmannsthal ("Hofmannsthal et son temps"), il assignera à la philosophie un "rang de subordination" par rapport à la littérature. Mais il changera son attitude dans ses œuvres postérieures, et plus particulièrement dans sa Psychologie des masses, de même que dans le deuxième tome de ses essais, dont la traduction française n'a pas encore été effectuée, mais dont Arendt nous livre quelques extraits qui s'orientent dans le sens de la théorie de la connaissance. Et où l'on voit, par le fait même, que Hannah Arendt a su contrevenir au caractère prescriptif d'une observation exposée par le jeune Kant sur le rapport des sexes

5 Hannah Arendt, *Introduction * dans Hermann Broch, Création littéraire et connaissance, Paris, Gallimard, coll. *Tel*, p. 7-43. 
(et énoncée dans un style disgracefully pretty): "En conséquence, la femme n'apprendra pas la géométrie " 6 .

Ce qu'il importe de cerner tout d'abord, dans ce retournement de l'attitude de Broch envers la philosophie, c'est-à-dire de la conception qu'il se faisait des rapports entre littérature et philosophie lorsqu'il affirmait que celle-ci devait "remettre la connaissance" à celle-là, c'est qu'il s'est dirigé progressivement d'une théorie des valeurs à une théorie de la vérité: "Il est surprenant de constater avec quelle fréquence Broch utilise dans ce contexte les mots contrainte, nécessité, nécessité contraignante, et comment il se reposait sur le caractère coercitif de l'argumentation logique" (p. 29). Mais ce qu'il faut surtout prendre ici en considération, c'est que mêrme à l'époque où il subordonnait la philosophie à la littérature, Broch avait su établir une distinction entre la connaissance comme activité de recherche logique et la pensée comme travail littéraire et philosophique. En somme, Hermann Broch le romancier des Somnambules n'a jamais proposé d'amalgamer littérature et philosophie dans une même armature, pas plus qu'Hermann Broch l'essayiste de la Psychologie des masses n'a cherché à promouvoir un syncrétisme entre la fiction romanesque et la théorie de la connaissance. Broch n'a donc jamais rêvé de mettre en place un quelconque dispositif d'intégration maximale amalgamant l'argumentation logique et le lyrisme poétique, pas plus qu'il n'avait voulu que la philosophie se fasse "avaler " par la littérature (à moins de confondre activité cognitive et activité digestive): tout au plus, que la première "remette la connaissance "à la deuxième, ce qui n'est pas la même chose. Et comme Hannah Arendt l'a rappelé à la toute fin de son introduction: "La mission dont Broch parle si fréquemment [...] n'est, en dernière analyse, de nature ni logique ni liée à la théorie de la connaissance, bien qu'il retrouve partout cette mission dans la logique et dans la théorie de la connaissance et qu'il se serve de celles-ci pour le démontrer. Cette mission est l'exigence éthique [...] le droit des hommes à l'assistance "(p. 43). C'est précisément cette éthique de la responsabilité que Scarpetta n'a de cesse de réduire à une technique romanesque dans sa version du postmodernisme qui, comme on le voit, relève de la fiction théorique.

Emmanuel Kant, Observations sur le sentiment du beau et du sublime, Paris, GF-Flammarion, 1990, p. 123. 


\section{De l'excessif Georges Bataille à l'imprésentable Jean-François Lyotard}

Avec la publication de La condition postmoderne ${ }^{7}$, JeanFrançois Lyotard est incontestablement devenu l'agent officiel du postmodernisme parisien: parce que c'est lui qui a importé le concept des États-Unis, bien que dans le transfert d'un continent à l'autre, la définition même du concept a pris l'allure d'un trafic d'influence transatlantique. Et puisque c'est la discussion qui l'a opposé à Jürgen Habermas qui a reçu le plus d'attention de la part des divers commentateurs de "l'affaire", la parution du Postmoderne expliqué aux enfants ${ }^{8}$ et du Discours philosophique de la modernité 9 sont à retenir pour le bilan comptable.

D'abord il convient de souligner que chez Lyotard, philosophe de formation, les rapports entre la philosophie et la littérature ne sont pas considérés dans les mêmes termes que chez Scarpetta. Ou plus précisément, il n'y a pas, dans sa problématique, de postulat d'une quelconque prééminence d'une discipline par rapport à l'autre. Lyotard traite le phénomène littéraire du point de vue de l'esthétique kantienne, ce qui ne signifie pas qu'il le fait en fonction du rationalisme néo-kantien, à la manière, par exemple, d'Ernst Cassirer dans La philosophie des Lumières ${ }^{10}$ : il procède plutôt par incursion du territoire romanesque à partir d'une extrapolation de l'esthétique du sublime. Ainsi, pour induire les procédés littéraires de certains écrivains, il écrira, dans "Réponse à la question: qu'est-ce que le postmoderne?" (extrait du Postmoderne expliqué aux enfants):

L'œuvre de Proust et celle de Joyce font l'une et l'autre allusion à quelque chose qui ne se laisse pas rendre présent. L'allusion [...] est peut-être un tour d'expression indispensable aux œuvres qui relèvent de l'esthétique du sublime. Chez Proust, ce qui s'élude pour payer le prix de cette allusion, c'est l'identité de la conscience en proie au trop de temps. Mais chez Joyce c'est l'identité de l'écriture en proie au trop de livres ou de littérature (p. 31).

7 Jean-François Lyotard, La condition postmoderne, Paris, Minuit, 1979.

8 Jean-François Lyotard, Le postmoderne expliqué aux enfants, Paris, Galilée, 1986.

9 Jürgen Habermas, Le discours philosophique de la modernité, Paris, Gallimard, coll. *NRF*, 1988.

10 Ernst Cassirer, - Les problèmes fondamentaux de l'esthétique •, dans $\mathrm{La}$ philosopbie des Lumières, Paris, Fayard, coll. *Agora ${ }_{2} 1966$, p. 351-445. 
Et ce motif subira un développement tout au cours d'un long paragraphe où vont s'aligner les phrases voulant que "Proust allègue l'imprésentable au moyen d'une langue intacte dans sa syntaxe et son lexique "bien que l'institution littéraire dont Proust hérite soit "subvertie" puisque "le héros n'est pas un personnage mais la conscience du temps", ou encore que "l'identité de l'écriture avec elle-même à travers le dédale de l'interminable narration suffit à connoter cette unité, qu'on a pu comparer à celle de la Pbénoménologie de l'esprit" alors que "Joyce fait deviner l'imprésentable dans son écriture même, dans le signifiant . Sans compter que chez Joyce "la grammaire et le vocabulaire de la langue littéraire ne sont plus acceptées comme des données, ils apparaissent plutôt comme des académismes, des rituels issus d'une piété (comme disait Nietzsche) qui empêche que l'imprésentable soit allégué " (p. 32).

Par cette façon de procéder, Jean-François Lyotard, plutôt que d'amener une discipline à en "avaler "une autre (et risquer, comme Scarpetta, de confondre l'indigence et l'indigeste), cherche au contraire à dissocier le jugement de goût du jugement de connaissance (pour parler à partir des catégories kantiennes de la (ritique du jugement), mais avec des conséquences dont on peut penser qu'elles sont aussi inquiétantes qu'étranges, pour les philosophes comme pour les écrivains. Pour s'en convaincre, il suffit d'examiner la façon dont le philosophe allemand Jürgen Habermas analyse le cas de figure d'un des écrivains qui aura eu le plus d'influence au sein de la tendance poststructuraliste, tendance dont on retrouve les sédiments dans le postmodernisme de Lyotard ${ }^{11}$. Avec un essai intitulé "Entre érotisme et économie générale: Bataille "(extrait du Discours philosopbique de la modernitê), Habermas propose une tout autre manière de faire de la philosophie en parlant de la littérature. Pour expliquer les apories de la trajectoire intellectuelle qui amènera Bataille à écrire à la fois comme écrivain surréaliste (puis ex-surréaliste) et comme émule de Hegel puis de Nietzsche, Habermas montre que "chaque fois, notamment, qu'il s'efforce par la science et la philosophie de parvenir à des aperçus réflexifs ayant une force pratique telle qu'elle transforme des personnes vaguement

11 Cf. Pierre Milot, .Structuro-marxisme et post-structuralisme •, dans Le paradigme rouge, Candiac, Éditions Balzac, coll. "Littératures à l'essai ", 1992, p. 211-270. 
concernées en parties prenantes de soi, Bataille se laisse glisser imperceptiblement dans le sillage d'une dialectique de la raison" (p. 279). De même, dira Habermas, si «à la fin de sa vie, Bataille s'aperçoit que sa double carrière d'écrivain et de philosophe lui ménage la possibilité de se retirer avantageusement de la philosophie et de la science ", c'est que "l'érotisme le conduit à penser que la connaissance de l'essentiel est réservée à une expérience mystique, au silence, les yeux clos, et que la connaissance discursive reste désespérément enfermée dans le cercle des séquences linguistiques" (p. 280). Or, constate le philosophe allemand, c'est par cela même que Bataille renonce à la part théorique de son entreprise (qu'il n'a pourtant jamais cessé de revendiquer, même pour son essai sur L'érotisme): la philosophie ne pouvant se permettre une attitude aussi débridée à l'égard du langage, sauf à s'éliminer comme discipline ${ }^{12}$. Évidemment, on pourrait ici reprocher à Habermas de perdre de vue que le corps a ses pulsions que la raison ignore. Mais ce serait tomber au niveau de la raison priapique d'un Lucien Francour, le chantre de la poésie libidinale: "Baisse tes culottes, montre-moi ton vrai visage ${ }^{13}$. Ou alors, on pourrait confronter Habermas à la raison égérique d'un Sören Kierkegaard, le scripteur de la séduction philosophique: *Libre aux disputailleurs savants de mettre de l'orgueil à éviter toute contradiction; la vie d'une jeune fille est trop riche pour en être exempte et elle rend donc la contradiction nécessaire " ${ }^{14}$. Mais puisque dans toute cette affaire il s'agit de dépasser la controverse métaphysique entre corps et esprit, c'est à partir de la triade des sphères kantiennes de la raison moderne (connaissance, éthique, esthétique) que Jürgen Habermas se propose d'éclairer la posture du corps (dressed to kill) dans le boudoir obscur de la modernité.

Si Bataille écrit que "la philosophie utilise le langage de manière que jamais le silence ne lui succède "et que par conséquent "le moment suprême excède nécessairement l'interrogation philosophique", c'est qu'il considère que la philosophie est nécessairement excédée par la littérature: de sorte que la philo-

12 Là-dessus, voir Jacques Bouveresse, Le pbilosopbe chez les autophages, Paris, Minuit, coll. «Critique*, 1984.

13 Lucien Francour, Snack bar, Montréal, Les Herbes rouges, $n^{\circ} 10,1973$, p. 13.

14 Sören Kierkegaard, Le journal du séducteur, Paris, Gallimard, coll. «Folio Essais *, 1989, p. 145. 
sophie ne servirait qu'à imposer à cette dernière les contraintes de la raison. C'est la même contradiction performative que rencontre Lyotard lorsque, partant d'une esthétique du sublime, il cherche à faire la promotion d'un postmodernisme littéraire et philosophique axé sur une "combinaison intrinsèque de plaisir et de peine" excédant la dialectique de la raison. Invoquant "le plaisir que la raison excède toute présentation * et "la douleur que l'imagination ou la sensibilité ne soient pas à la mesure du concept ", il refuse l'impératif axiologique que sa discipline présuppose chez tout professionnel de la connaissance: l'exigence de validité intersubjective ${ }^{15}$. C'est précisément à l'égard de cette force d'obligation que Lyotard n'a de cesse de se montrer insouciant dans sa version du postmodernisme qui, comme on le voit, relève de la littéro-philosophie.

Paradoxalement, alors que Jean-François Lyotard se proposait de faire "subir" à Kant et à Hegel "le sévère réexamen que la postmodernité impose à la pensée des Lumières" (p. 16), Guy Scarpetta, qui dans la conclusion de L'impureté s'affairait à rappeler le rôle essentiel des écrivains français dans l'avènement d'une réflexion esthétique "ne visant pas a priori au détachement de l'objectivité conceptuelle" (p. 384), n'en déclarait pas moins: "Il m'est parfois arrivé, en écrivant ce livre, de ressentir un manque: celui (dans la tradition française, du moins) d'une réflexion esthétique d'envergure, de type de celles de Kant ou de Hegel dans la pensée allemande" (p. 382). Mais à lire Lyotard et Scarpetta on risque de rester en manque encore longtemps, "du moins dans la tradition française", tout au moins dans celle que fréquentent Scarpetta et Lyotard. Car ce qui a caractérisé la "réflexion esthétique d'envergure "dans la "pensée allemande ", outre son attachement à "l'objectivité conceptuelle", donc à la connaissance, c'est aussi l'envergure de sa réflexion éthique. Voilà pourquoi je n'écris pas d'essais postmodernes.

15 Sur le concept d'intersubjectivité, voir Karl-Otto Apel, L'éthique à l'âge de la science, Lille, Presses Universitaires de Lille, 1987. Et pour une analyse philosophique qui diverge à la fois de Lyotard et de Habermas concernant les rapports entre littérature et philosophie, voir Richard Rorty, "Déconstruction et circonvention", dans Science et solidarité. La vérité sans le pouvoir, Paris, L'éclat, coll. "Tiré à part”, p. 85-111. 